\title{
Technology Developments in Real-Time Tsunami Measuring, Monitoring and Forecasting
}

\author{
Christian Meinig, Scott E. Stalin, Alex I. Nakamura, Frank González \\ NOAA, Pacific Marine Environmental Laboratory (PMEL)
}

\author{
Hugh B. Milburn \\ Oceanographic Engineer
}

\begin{abstract}
Fast, accurate tsunami forecasts are an essential component of an effective tsunami warning system. Decision-makers at Tsunami Warning Centers must assess the hazard to coastal communities by rapidly collecting and interpreting earthquake and sea-level data. The stakes are high: A missed warning could devastate entire regions and needless evacuations are expensive, dangerous and erode confidence in the warning system. Tsunami forecasting technology under development at NOAA/PMEL is based on the welltested approach used in many other forecast systems-i.e., the integration of real-time measurement and modeling technologies. Real-time monitoring and measurement of sea-level data in the deep ocean is presently made by a seven-station network of DART (Deep-ocean Assessment and Reporting of Tsunamis) systems. DART II is a new generation system that will have additional features and capabilities to aid the forecasting ability of Tsunami Warning Centers. As a result of the devastating impact of the 26 December 2004 Sumatra tsunami and the proven value of the DART array, the number of network stations will be increased to 39 by mid-2007.
\end{abstract}

\section{INTRODUCTION}

This paper describes the system components that comprise a critical portion of NOAA's tsunami forecast, warning, and mitigation system. The components discussed include details of the second-generation Deep-Ocean Assessment and Reporting of Tsunamis (DART II) system, and gives an overview of the model based interpretations used to make tsunami forecasts. Tsunami data from the DART system can be combined with seismic data ingested into a forecast model to generate accurate tsunami forecasts for coastal areas [1]. Warning and guidance are one of the components that make an effective warning system and develop tsunami-resilient communities. Other critical and necessary components include: 1) Hazard Assessment, and 2) Mitigation; however, these components are beyond the scope of this paper.

The motivation for developing a transportable, realtime, deep ocean tsunami measurement system was to forecast the impact of tsunamis on coastal areas in time to save lives and protect property. Over the past 20 years, PMEL has identified the requirements of the tsunami measurement system through evolution in both technology and knowledge of deep ocean tsunami dynamics. The requirement for transportability was a conservative approach to a phenomenon that had little data to guide strategies for choosing deployment sites. The requirement for real-time transmissions was to provide data in time to create a forecast. The first-generation DART design featured an automatic detection and reporting algorithm triggered by a threshold wave-height value. The DART II design incorporates two-way communications that enables tsunami data transmission on demand, independent of the automatic algorithm; this capability ensures the measurement and reporting of tsunamis with amplitude below the autoreporting threshold.

For our purposes in this paper, when we refer to measured "sea level height," it is inferred from the seafloor pressure measurements by assuming a constant $1 \mathrm{psi}=$ $670 \mathrm{~mm}$ of water height. The errors resulting from neglecting variations in acceleration and density terms are not significant for tsunami detection, where only the relatively small changes are used in the analysis of wave characteristics.

\section{A. Reaction to the Indian Ocean Tsunami of December 26, 2004}

The 9.0 earthquake of December 26, 2004 and the resulting tsunami killed more than 300,000 people-more casualties than any other tsunami ever recorded (Fig. 1). It was the largest trans-oceanic tsunami in over 40 years. A second earthquake only 3 months later of magnitude 8.7 has instilled more fear in the survivors of the first disaster, and has everyone wondering when the next tsunami will occur [2], and how their coastal community can be made tsunami-resilient $[3,4]$.

As a result of this tragedy, there is strong interest in installing a global tsunami warning system as soon as possible. Additionally, the Bush Administration has provided funds to expand the current tsunami warning system by deploying 39 additional DART II systems in the Pacific, the Atlantic, and the Caribbean [5].

\section{TSUNAMI FORECASTING SYSTEM}

PMEL tsunami R\&D is focused on saving lives by improving the speed, accuracy and reliability of NOAA tsunami warnings. The strategy is to develop a fast, accurate operational tsunami forecast system to provide an optimal interpretation of the available earthquake and sea-level data and quantify the potential tsunami impact on coastal 


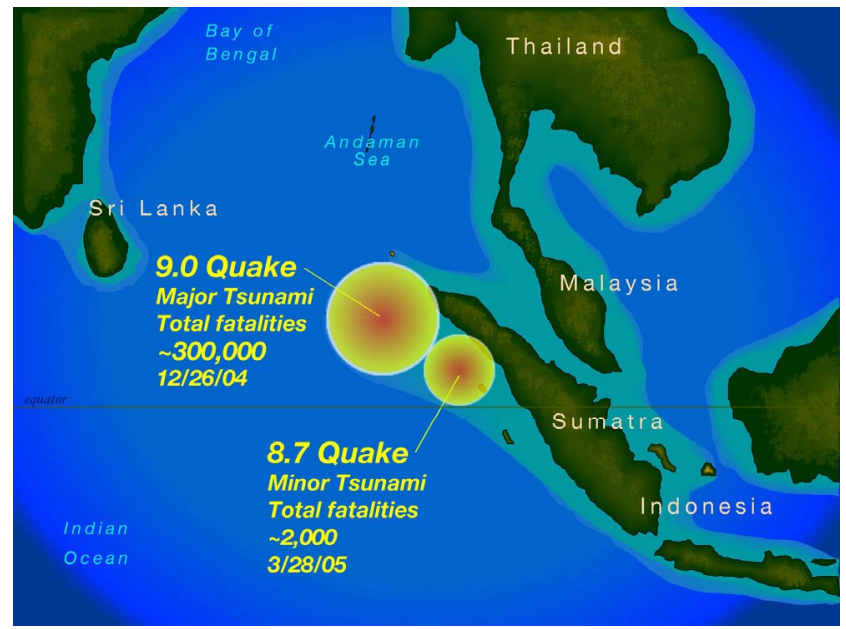

Fig. 1: Location of two earthquakes in the Indian Ocean: the first caused massive casualties; the second created panic.

communities. Such a system is critical to decision-makers at Tsunami Warning Centers, who must rapidly assess the hazard to coastal communities. The stakes are high: a missed warning could result in devastating fatalities; and needless evacuations are dangerous, expensive, and erode confidence in the warning system. Tsunami forecasting technology under development at NOAA/PMEL is based on the well-tested approach used in most hazard forecast systems - i.e., the integration of real-time measurement and modeling technologies.

DART systems, developed by PMEL, have proven to be robust and reliable. A seven-buoy array has been transitioned to operational status to the National Data Buoy Center (NDBC) and is monitoring and reporting water column heights in the Pacific Ocean at relevant locations for tsunami propagation. The cumulative data return ratio for the array from 1997-2003 exceeded $91 \%$. The data return ratio is computed by dividing the total data received on shore by the total expected data. The DART systems have reacted to and reported six seismic-induced wave events that contributed to operational decisions, avoiding false alarms and the resultant costs associated with them [6]. One incident, a magnitude 7.5 earthquake in the Aleutian Islands that occurred on November 17, 2003, triggered a tsunami "watch" in Hawaii and Alaska. Data from three tsunameters showed the wave was not significant, and no warning was issued, thus saving Hawaii $>\$ 68 \mathrm{M}$ in evacuation costs.

Currently, tsunami measurements are sparse, as the result of vast oceanic distances, the hostile ocean environment, and the associated logistical and maintenance difficulties. As future DART systems evolve to address these difficulties, the density of DART networks will increase; however, model-based interpretation and analyses of the data will always be needed to meet the operational standards required when lives and property are at stake.

\section{A. Most Model}

Model-based interpretation of the tsunami, such as those by the MOST (Method of Splitting Tsunamis) model occurs in two stages. First, a pre-computed database of deep ocean model simulations is exploited to formally invert the real-time tsunameter data stream and produce a linear best-fit solution in deep water; this step is completed within a few minutes of data acquisition. Second, the deep-water values just offshore are used to initiate realtime execution of non-linear inundation models to provide community-specific forecasts of tsunami inundation; this step is completed in less than $10 \min [1]$.

On November 17, 2003, a validating, comprehensive test was conducted of the capability to provide a real-time, community-specific forecast. A large earthquake generated a tsunami that was detected by three tsunameters (Fig. 2). A PMEL research scientist took the first forecast step, combining the real-time data with the MOST model

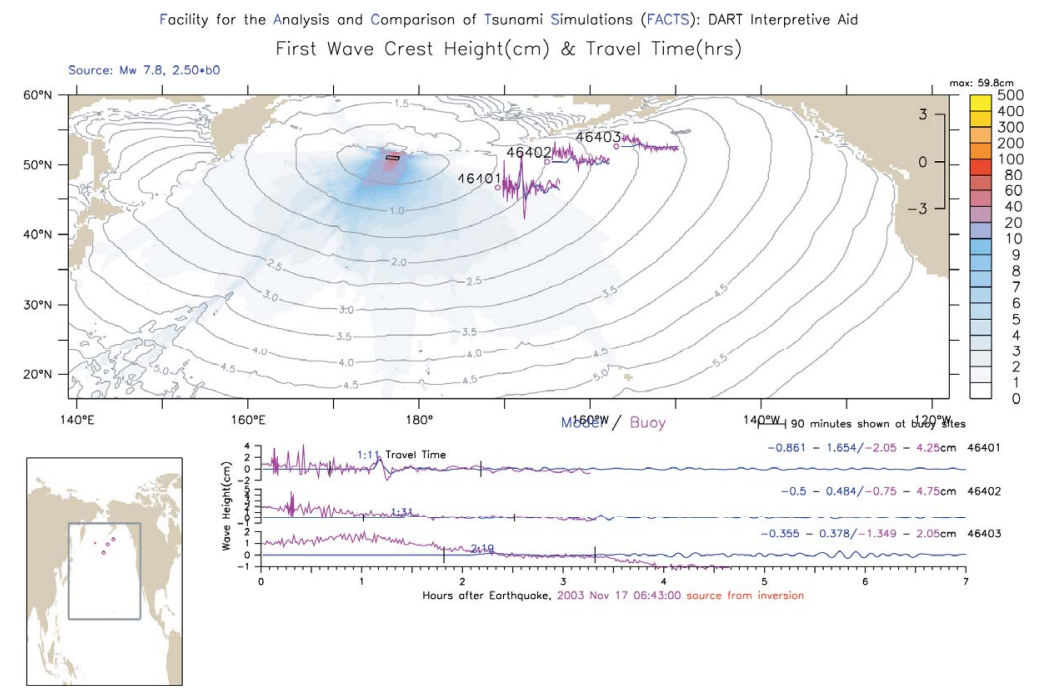

Fig. 2. Offshore forecast for the 2003 Rat Island tsunami. Top frame shows maximum computed wave heights (color contours), travel time contours in hours after the earthquake (solid lines), and locations of DART stations. Bottom frame shows reference map (left) and comparison of the model (blue) and tsunameter data (magenta) 


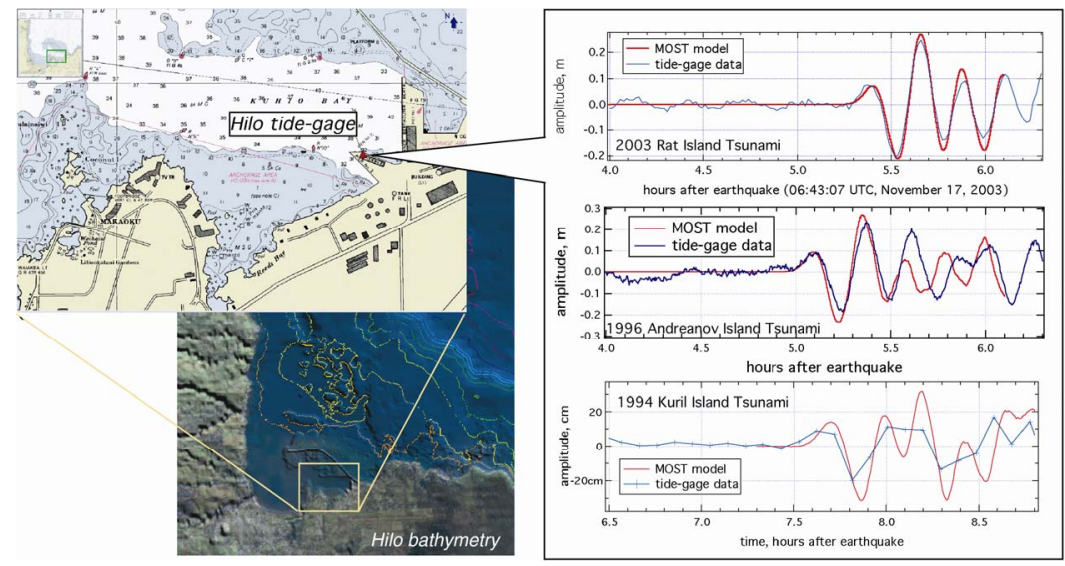

Fig. 3. Coastal forecast at Hilo, HI for 2003 Rat Is. tsunami (top), 1996 Andreanov Is. (middle) and 1994 Kuril Is. (bottom) tsunamis. Right frame shows a comparison of forecasted (red) and measured (blue) gage data. Left frame shows location of Hilo tide gage (top map) and digital elevation data for the high-resolution inundation computation (bottom).

database to produce expected tsunami wave heights at the three tsunameter locations. When the actual height values were reported from the 46401 DART station $\left(50^{\circ} \mathrm{N}\right.$ $\left.171^{\circ} \mathrm{W}\right)$, the model was quickly adjusted and correctly predicted tsunami time series values at the two other DART locations. (It is significant that this tsunami data inversion also produced a better estimate of the actual earthquake magnitude, Mw 7.7-7.8, as later confirmed by USGS and Harvard Seismology Group.)

The second forecast step was then taken; a fineresolution, nonlinear inundation model was initiated with the predicted offshore values, and a simulation was completed to produce a tsunami forecast for Hilo, Hawaii, before the waves had reached the community. As predicted, Hilo inundation did not occur. And later comparison of the forecast and observed time series at the Hilo tide gage produced a remarkable correspondence of the waveforms for several cycles of the wave, characterized by a maximum amplitude of $25 \mathrm{~cm}$ (Fig. 3). The significance of this result is that, although a number of hindcasts of historical data have been performed, and although the result was not an official, operational product, the "blind" forecast was produced in real-time as the event unfolded; the accuracy of the result was a convincing, "proof of concept" demonstration that the methodology can provide reliable, sitespecific, real-time forecasts.

A DART capability that is critically important to the accuracy and reliability of the forecast is the acquisition of tsunami measurements in the deep ocean, rather than at coastal port or harbor sites; this allows interception of the tsunami as it propagates from the source region to distant coastlines, and eliminates complex coastal interactions that degrade the accuracy of the data inversion. In addition, the DART systems meet a number of data stream requirements that are essential to an operational tsunami forecast system:
1. Measurement:
tsunami amplitude time series
2. Accuracy:
$0.5 \mathrm{~cm}$ or less
3. Sampling:
$1 \mathrm{~min}$ or less

\section{Processing: 2 min or less \\ 5. Delivery: $\quad 5 \mathrm{~min}$ or less}

For these reasons, a recent NOAA review of nine advanced measurement techniques found that, among these nine, DART systems are “ ... currently the most effective means of detection of a tsunami" [7].

\section{DART II SYSTEM}

A DART II system, shown in Fig. 4, consists of two physical components: a tsunameter on the ocean floor and a surface buoy with satellite telecommunications capability. The DART II systems have bi-directional communication links and are thus able to send and receive data from the Tsunami Warning Center and others via the Internet. The web site for the DART data is supported by NDBC and can be seen at: http://www.ndbc.noaa.gov/dart.shtml.

\section{A. Deep-Ocean Pressure Measurements}

Tsunamis have wave lengths of hundreds of kilometers, and are considered shallow water waves. They "feel" the bottom in deep water, and increase in height only as they shoal in areas of decreasing depth. Wind waves are called "deep water waves" where they have negligible impact below half of their wave length. This well-studied exponential decay of orbital motion with depth was derived and presented by George Stokes in 1847. This phenomenon makes the deep ocean an ideal low-pass filter, and allows tsunamis, tides, and other long-period events to be detected by simply measuring the pressure at a fixed point on the seafloor.

The earthquake waves shown in Fig. 5 are an interesting feature of tsunami monitoring with bottom pressure sensors. Earthquake waves travel significantly faster than tsunami waves, and frequently trip the tsunameter into "Event Mode" before the tsunami arrives. The vertical shifting of the seafloor from the earthquake acts to lift or 


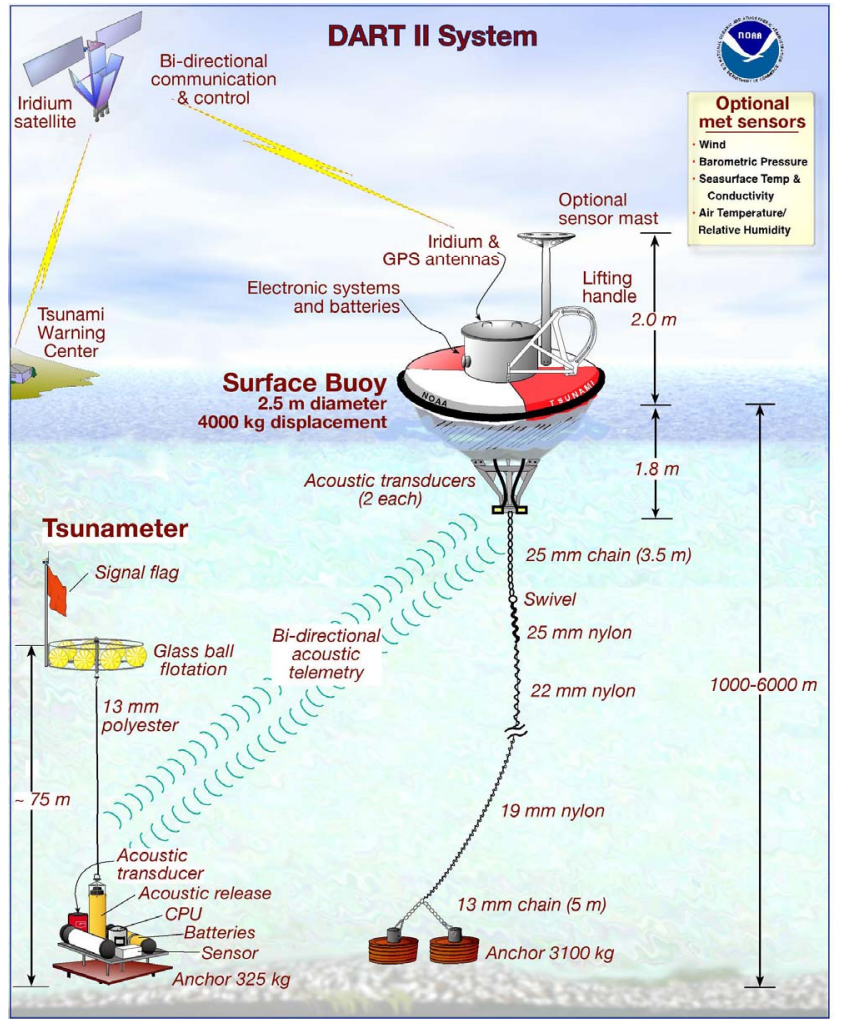

Fig. 4: DART II system.

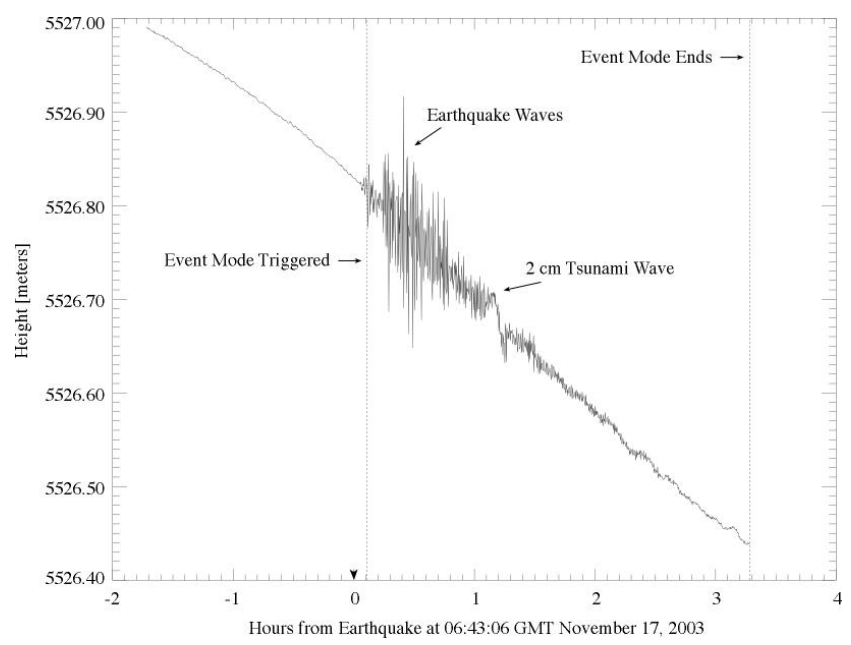

Fig. 5: Tsunami of November 17, 2003, as measured at the tsunameter located at $50^{\circ} \mathrm{N}, 171^{\circ} \mathrm{W}$.

compress the water column above, showing an increase in pressure as the seafloor rises, or decrease in pressure as the seafloor falls.

The relatively quiet environment on the seafloor in deep water can be used to validate the performance of pressure measuring systems. Observed vs. predicted tidal variations can be used to validate the gross measurement, and the finest resolution can be seen by the sample-tosample variation at periods of high and low tide. The low noise and high resolution in the tsunameters have pre- cluded false alarms from occurring on the deployed DART systems.

\section{B. DART II Characteristics}

DART II performance characteristics are summarized in Table 1. These performance characteristics helped to drive the research and development of the DART II system. Specific engineering details about the tsunameter and the buoy follow.

TABLE 1

DART II PERFORMANCE CHARACTERISTICS

\begin{tabular}{ll}
\hline Characteristic & Specification \\
\hline Reliability and data return ratio: & Greater than $80 \%$ \\
Maximum deployment depth: & $6000 \mathrm{~m}$ \\
Minimum deployment duration: & Greater than 1 year \\
Operating Conditions: & Beaufort 9 (survive \\
& Beaufort 11) \\
Maintenance interval, buoy: & Greater than 2 years \\
Maintenance interval, tsunameter & Greater than 4 years \\
Sampling interval, internal record: & 15 sec \\
Sampling interval, event reports: & 15 and 60 sec \\
Sampling interval, tidal reports: & 15 min \\
Measurement sensitivity: & Less than 1 mm in \\
& 6000 m; $2 \times 10^{-7}$ \\
Tsunami data report trigger & Automatically by \\
& tsunami detection \\
& algorithm; on- \\
& demand, by warning \\
& center request \\
Reporting delay: & Less than 3 min \\
Maximum status report interval: & Less than 6 hrs \\
\hline
\end{tabular}

\section{Tsunameter}

The block diagram in Fig. 6 shows how the components of a tsunameter function together, and an ocean recovery of a tsunameter is shown in Fig. 7. The computer reads pressure readings, runs a tsunami detection algorithm, and sends and receives commands and data to and from the buoy via an acoustic modem.

The DART II pressure sensor is a $0-10,000$ psi model $410 \mathrm{~K}$ Digiquartz ${ }^{\circledR}$ unit manufactured by Paroscientific, Inc. The transducers use a very thin quartz crystal beam, electrically induced to vibrate at its lowest resonant mode. The oscillator is attached to a Bourdon tube that is open on one end to the ocean environment [8]. The pressure sensor outputs two frequency-modulated square waves, proportional to the ambient pressure and temperature. The temperature data is used to compensate for the thermal effects on the pressure-sensing element. 


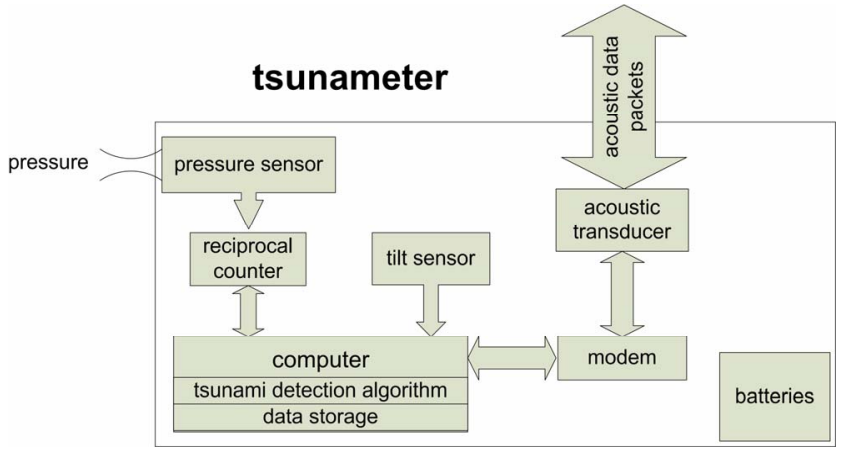

Fig. 6: Block diagram of DART II Tsunameter.

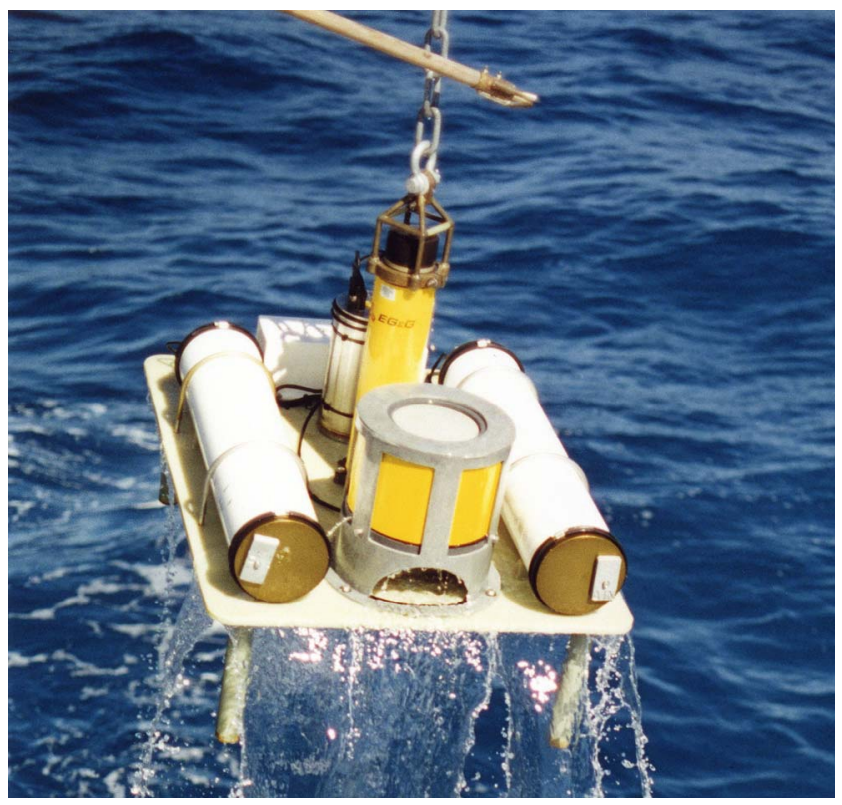

Fig. 7: Tsunameter recovery.

\section{1) Reciprocal Counter and Computer}

The high-resolution precision reciprocal counting circuit continuously measures the pressure and temperature signals simultaneously, integrating them over the entire sampling window, nominally set to $15 \mathrm{sec}$. There is no dead period between the sampling windows. The circuit has a sub-millimeter pressure and sub-millidegree temperature least-count resolution. The reference frequency for the reciprocal counter is derived from a low power, very stable, $2.097152 \mathrm{MHz}$, temperature-compensated crystal oscillator. A real-time calendar-clock in the computer also uses this reference for a time base. At the end of each sampling window the computer reads the pressure and temperature data and stores the data in a flash memory card. A 15 -sec sampling period generates about 18 megabytes of data per year.

The embedded computer system in both the buoy and the tsunameter was designed around the 32-bit, 3.3 volt Motorola 68332 microcontroller, and was programmed in
C. It was built to be energy efficient for long-term battery powered deployment. The computer has $4 \mathrm{Mb}$ of flash memory, a 12-bit A/D converter with eight input channels, two RS232 channels, a hardware watchdog timer, a realtime clock, and 512 bytes of RAM. The embedded computer implements and regulates the primary functions of the surface and seafloor units: transmitting data communications, running the tsunami detection algorithm, storing and retrieving water column heights, generating checksums, and conducting automatic mode switching.

\section{2) Acoustic Communications}

A Benthos ATM-880 Telesonar acoustic modem with an AT-421LF directional transducer has a $40^{\circ}$ conical beam which is used to transmit data between the tsunameter and the surface buoy. Modems transmit digital data via MFSK modulated sound signals with options for redundancy and convolutional coding. Transducers are baffled to minimize ambient noise from entering the receiver. The acoustic modems are configured to operate in the 9$14 \mathrm{kHz}$ frequency band at $600 \mathrm{baud}$, using MFSK and error-correcting coding. The source level is at $193 \mathrm{~dB}$ re $1 \mu \mathrm{Pa} @ 1 \mathrm{~m}$ with a $40 \mathrm{VDC}$ supply.

The communication uses a modified x-modem protocol. Entire packets of data with many blocks are sent without requesting an acknowledgement from the receiver after each block. Missing or erroneous blocks are requested to be resent again as individual blocks. If the system is unable to connect, a maximum of two retries are attempted. Most importantly, the modified x-modem protocol greatly reduces power consumption, and efficiently supports high data throughput and integrity.

\section{3) Detection Algorithm and Reporting Modes}

Each DART II tsunameter is designed to detect and report tsunamis autonomously [9]. The Tsunami Detection Algorithm works by first estimating the amplitudes of the pressure fluctuations within the tsunami frequency band, and then testing these amplitudes against a threshold value. The amplitudes are computed by subtracting predicted pressures from the observations, in which the predictions closely match the tides and lower frequency fluctuations. If the amplitudes exceed the threshold, the tsunameter goes into Event Mode to provide detailed information about the tsunami.

Tsunameters operate in one of two data reporting modes: A low power, scheduled transmission mode called "Standard Mode" and a triggered event mode simply called "Event Mode."

Standard Mode reports once every 6 hours. Information reported includes the average water column height, battery voltages, status indicator, and a time stamp. These continuous measurements provide assurance that the system is working correctly. 


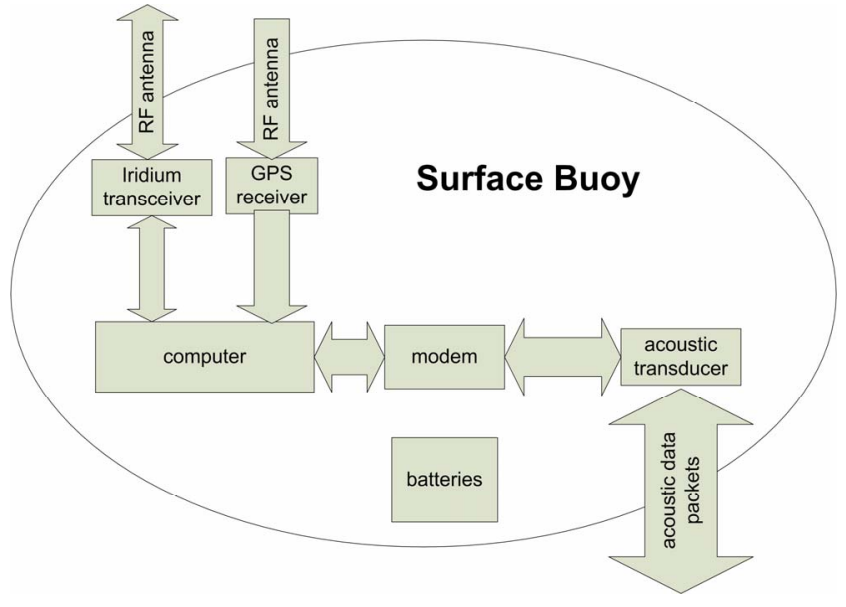

Fig. 8: Block diagram of DART II surface buoy.

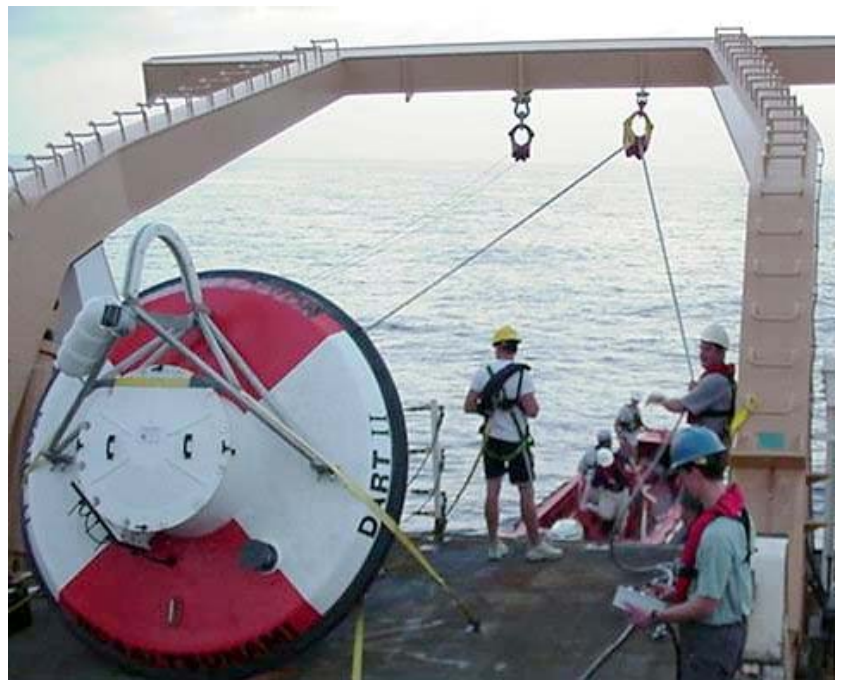

Fig. 9: DART II buoy ready for deployment on Hi'ialakai in 2005.

Event Mode reports events such as earthquakes and /or tsunamis when a detection threshold is exceeded. The Tsunami Detection Algorithm triggers when measured and predicted values differ by more than the threshold value. Waveform data are transmitted immediately (less than a 3-min delay).

Tsunami waveform data continue to be transmitted every hour until the Tsunami Detection Algorithm is in a non-triggered status. At this point the system returns to the Standard Mode.

\section{4) Batteries}

The tsunameter computer and pressure measurement system uses an Alkaline D-Cell battery pack with a capacity of 1560 watt-hours. The acoustic modem in the tsunameter is powered by similar battery packs that can deliver over 2,000 watt-hours of energy. These batteries are designed to last for 4 years on the seafloor; however, this is based on assumptions about the number of events that may occur and the volume of data request from the shore. Bat- tery monitoring is required to maximize the life of the system.

\section{Surface Buoy}

The DART II surface buoy, shown in Figs. 8 and 9, relays information and commands from the tsunameter and the satellite network. The buoy contains two identical electronic systems to provide redundancy in case one of the units fails. The computer is the same type used in the tsunameter and it processes messages from both the satellite and the tsunameter. The Benthos Telesonar acoustic modems and transducers are the same as used in the tsunameter. The downward-looking transducers are mounted on the buoy bridle at a depth of $1.5 \mathrm{~m}$ below the sea surface. A multi-layered baffle system of steel, lead, and syntactic foam shields the transducers from noise, and cushions them with rubber pads for a soft mount.

The Standard Mode transmissions are handled by both electronic systems on a preset schedule. The Event Mode transmissions, due to their importance and urgency, are immediately transmitted by both systems simultaneously.

The surface mooring uses a $2.5 \mathrm{~m}$ diameter fiberglass over foam disk buoy with a displacement of $4000 \mathrm{~kg}$. The mooring line is $19 \mathrm{~mm}$ eight-strand plaited nylon line with a rated breaking strength of $7100 \mathrm{~kg}$, and is deployed to maintain a tight watch circle, keeping the buoy positioned within the cone of the acoustic transmission. In temperate areas where fish tend to aggregate and bite lines, wire rope is use on the upper few hundred meters of the mooring.

\section{1) Iridium Transceiver}

A Motorola 9522 L-Band Iridium transceiver from NAL Research provides data connectivity via the Iridium Satellite Network. The buoy computer connects to the transceiver using an RS232 serial port. Data is transferred at 2400 baud similarly to the familiar dial-up modem connections. A typical Standard Mode report takes approximately $30 \mathrm{sec}$, including the time it takes to complete the connection, transmit the data, and disconnect.

A Leadtek model 9546 GPS receiver is used to maintain the buoy's computer clock's accuracy to within $\sim 1 \mathrm{sec}$ of GMT. Additionally, a GPS position is reported once per day to monitor buoy location.

\section{2) Batteries}

The buoy's fiberglass well houses the system electronics and power supply, which is made up of packs of D-Cell alkaline batteries. The computer and Iridium transceiver are powered by 2,560 watt-hour batteries; the acoustic modem is powered by 1,800 watt-hour batteries. These batteries will power the buoy for at least 2 years.

The buoy is designed to mitigate the potentially dangerous build up of hydrogen gas that is naturally vented from alkaline cells. Design features include: 1) hydrogen 
getters (such as those from HydroCap Corp.); 2) pressure relief valves; and 3) spark-free components such as fiberglass or plastic.

\section{3) Data Communications}

A DART II innovation is the ability to send messages from a workstation on land to the buoy and the tsunameter. This bi-directional communication enables commands to be sent to the DART II system. The Tsunami Warning Center issues commands that are queued in a server until the DART II buoy is in Listen Mode, which operates on a $20 \%$ duty cycle. The redundant systems will turn on their respective Iridium transceivers at alternate times for $3 \mathrm{~min}$ out of $15 \mathrm{~min}$. This yields a maximum inaccessibility of only $6 \mathrm{~min}$. This scheme is employed to control the buoy power requirements by decreasing the standby power draw of the Iridium transceivers.

Data from each DART II system is downloaded and stored on a server via the Iridium Gateway and RUDICS server. The Warning Centers monitor this data stream in real time and are responsible for issuing warnings. In addition, the data is posted to a web server, and can be viewed in real time on the Internet.

\section{SUMMARY}

This paper describes aspects of the NOAA Tsunami Forecasting System, composed of two primary components - the DART II measurement system and the MOST modeling system. In the years since its initial funding in 1996, many lessons have been learned, and progress has been steady in five areas: tsunameters, moorings, telecommunications, software algorithms, and forecast modeling. These scientific and engineering advances at NOAA's Pacific Marine Environmental Laboratory (PMEL) have led to a highly-reliable measurement system that acquires and delivers tsunami data from deep ocean locations between the tsunami generating event and distant at-risk communities, and transmits these data in near real time to Tsunami Warning Centers and the Internet. The forecasting methodology employs the MOST numerical model to ingest and formally invert the real-time, DART water height values to provide site- and event- specific forecasts; this methodology has been tested and validated through numerous hindcasts of historical data and, most significantly, through a real-time, "blind forecast" conducted during the 17 November 2003 event. New technologies and methodologies are envisioned and must be studied and exploited where possible to provide improved warning guidance for tsunami hazards. The recent events in the Indian Ocean are a stark reminder that the value of these efforts should not be underestimated.

\section{ACKNOWLEDGMENTS}

We express our appreciation to the numerous individuals that contributed to the DART II and MOST develop- ments, in particular the leadership and guidance of Dr. Eddie Bernard. This work was jointly supported by NOAA and the National Tsunami Hazard Mitigation Program (NTHMP). This is PMEL Contribution No. 2836.

\section{REFERENCES}

[1] V.V. Titov, F.I. González, E.N. Bernard, M.C. Eble, H.O. Mofjeld, J.C. Newman, and A.J. Venturato, "Real-time tsunami forecasting: Challenges and solutions," Nat. Hazards, vol. 35(1), Special Issue, U.S. National Tsunami Hazard Mitigation Program, pp. 41$58,2005$.

[2] http://ioc.unesco.org/indotsunami/index.htm, "Towards a tsunami warning system in the Indian Ocean," 2005.

[3] E.N. Bernard, "Tsunami: Reduction Of Impacts through three Key Actions (TROIKA);" Proceedings of the International Tsunami Symposium 2001 (ITS 2001) (on CD-ROM), Session 1-1, Seattle, WA, 7-10 August 2001, pp. 247-262.; http://www.pmel.noaa. gov/its2001/Separate_Papers/1-01_Bernard.pdf, 2001.

[4] E.N. Bernard, (ed.), Developing Tsunami-Resilient Communities - The National Tsunami Hazard Mitigation Program, Springer, The Netherlands (ISBN 14020-3353-2) 184 pp., 2005.

[5] W.A. Morrissey, CRS Report for Congress; "Tsunamis: Monitoring, detection, and early warning systems," Resources, Science, and Industry Division; http://fpc.state.gov/documents/organization/46407.pdf, 2005.

[6] F.I. González, E.N. Bernard, C. Meinig, M.C. Eble, H.O. Mofjeld, and S. Stalin, "The NTHMP tsunameter network," Nat. Hazards, vol. 35(1), Special Issue, U.S. National Tsunami Hazard Mitigation Program, 25-39.; http://www.pmel.noaa.gov/tsunami/Dart/Pdf/gonz2663 .pdf, 2005.

[7] A.J. Gasiewski, A.G. Voronovich, O.A. Godin, G.L. Mader, S.I. Gutman, and M. O'Neil Baringer, "Advanced techniques for tsunami detection and hazard mitigation," Internal NOAA Review Document, May 3, 2005 .

[8] M. Yilmaz, P. Migliacio, and E.N. Bernard, "Broadband vibrating quartz pressure sensors for tsunameter and other oceanographic applications," Paroscientific, Inc. Redmond, WA USA; http://www.paroscientific. com/pdf/realtimetsunami.pdf, 2004.

[9] http://www.pmel.noaa.gov/tsunami/tda documentation.html, "Tsunami detection algorithm." 